$(22 \%)$. Over $17 \%$ of injuries resulted in death after hospitalization. These findings provide empirical bases for future studies. More research is needed to identify injury related factors useful for planning effective prevention strategies.

Conclusion The study was conducted with the goal of providing the baseline data to policy makers and other stakeholders to help guide future research, policy and funding agendas.

\section{TBI/Concussion injuries}

\section{PEDIATRIC HEAD INJURIES TREATED IN CHILDREN'S EMERGENCY DEPARTMENT FROM CHISINAU}

${ }^{1}$ A Cazacu-Stratu, ${ }^{1} \mathrm{~S}$ Cociu, 'S Cebanu, A Holostenco ${ }^{2}$. 'Nicolae Testemitanu State University of Medicine and Pharmacy of the Republic of Moldova, Chisinau, the Republic of

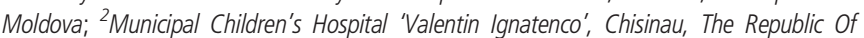
Moldova

10.1136/injuryprev-2021-SAVIR.61

Statement of purpose The purpose of this study was to explore the particularities of TBI among children in the Republic of Moldova.

Methods/Approach A prospective study was conducted among children patients with TBI at the Republican Municipal Children's Hospital 'Valentin Ignatenco'. We used data from the pilot TBI registry, from March, 1 - August 31, 2019. Data were uploaded using an electronic data collection tool - Red Cap and analyzed through Microsoft Excel, Epi Info 7. Data collection was performed by resident neurosurgery and a scientific researcher. The ethics committee's approval was obtained.

Results There have been identified 167 cases of TBI in children aged between 0-17 years old, of which 109 (65,3\%) male and $58(34,7 \%)$ female; most cases being in the age group 0-6 years old $59(35,3 \%)$, followed by a group of 10-14 years old $48(28,7 \%)$. Mostly, the injury appears in urban area 161 $(96,4 \%)$. The major cause- falling $97(63,6 \%)$ and the most affected group- 0-6 years old $44(26,3 \%)$. In the second place, there were road accidents $50(16,4 \%)$ with the most cases in the age group of 10-14 years old 16 (9,6\%), followed by assault/violence 19 (18,7\%). Most injuries occurred at home 45 $(26,9 \%)$, followed by transport area $41(24,6 \%)$, school education area $24(14,4 \%)$. Most cases registered in March 35 (21\%), followed by June and August with 29 (17,4\%), between 10.00- 18.00 (65,3\%), 20.00-22.00 (16,2\%).

Conclusion Data shows the most vulnerable age group, 0-6 years old, and 10-14 years old. The main mechanisms of TBI are falls and road accidents. The results of the study impose to develop prevention measurements for this target group.

Significance The main results obtained in the research will be included in the implementation of prevention actions and to development of specific activities for children.

\section{Occupational Safety}

\section{CROSS-SECTIONAL STUDY - THE PREVALENCE AND EFFECTS OF WORKPLACE VIOLENCE AGAINST MEDICAL STAFF IN THREE HOSPITALS OF TBILISI, GEORGIA}

M Kareli, N Pitskhelauri. Ivane Javakhishvili Tbilisi State University, Tbilisi, Georgia
Statement of purpose Violence at work is present at all work environments however, healthcare workers are at greater risk and the vast majority of cases go unreported. Existing scientific literature reveals that Workplace Violence (WPV) in health sector is very problematic in developing and transitional countries. Georgia as a developing country faces loads of human rights violation, including workplace violence, especially in the health care sector, however at present time there are no accurate and reliable data available. The main aim of the study was to assess prevalence, magnitude, consequences and potential risk factors of WVP against Medical Staff in three hospitals of Tbilisi.

Methods/Approach The cross-sectional study was conducted from May 2020 to August 2020 in three hospitals of Tbilisi. We used adapted and translated version of "Workplace violence in the health sector country case studies research instruments survey questionnaires'. After proving validity of the questionnaire, we distributed its online version. Statistical Analysis was performed in IBM SPSS Statistics 23. Fisher's Exact Test was used for finding associations

Results A total study population included 80 medical staff from three different hospitals of Tbilisi, Georgia. Medical staff had experienced different types of WPV, including: Verbal Threat or assault $61,5 \%$, physical assault $-7.5 \%$, sexual harassment $-2.5 \%$ and sexual assault- $1.5 \%$. $41 \%$ of study participants considered violence as part of their job and 29\% considered as part of their profession.

Conclusions Our study has indicated overall workplace prevalence against healthcare workers to be high in three hospitals of Tbilisi, Georgia.

Significance Violence is preventable, first step in violence prevention is recognizing that WPV is real and is one of the safety and health hazards. Guidelines for preventing and managing WPV are essential, each hospital should have mandatory violence reporting system and violence prevention policy, medical staff should not feel that violence is tolerable.

\section{Health equity}

\section{MENTAL HEALTH IN FORMERLY INCARCERATED BLACK MEN: A SYSTEMATIC MIXED STUDIES REVIEW}

H Addison, SF Jacoby. University of Pennsylvania School of Nursing, Philadelphia, USA

\subsection{6/injuryprev-2021-SAVIR.63}

Statement of purpose The disproportionate incarceration rate of Black men in the United States (US) is associated with a multiplicity of individual and community-level health outcomes. This review aims to evaluate the state of scientific knowledge on the mental health of Black men who have experienced incarceration and identify how Black men released from incarceration describe their mental health upon re-entry.

Methods/Approach This systematic mixed studies review used a sequential explanatory design incorporating quantitative and qualitative research. In June 2020, PubMed, CINAHL, PsycINFO, Social Work Abstracts, and Criminal Justice Abstracts were systematically searched. Of 538 articles identified, 22 met inclusion criteria as peer-reviewed original research, published in English from (2010-2020), relevant to the mental health of formerly incarcerated Black men in the US. 